\title{
COLLOQUE INTERNATIONAL «POLITICAL ECONOMY AND NATIONAL REALITIES PATTERNS AND PATHS IN THE ORIGINS AND DEVELOPMENT OF ECONOMIC SCIENCE» Fondation Luigi Einaudi Turin, 10-12 septembre 1992
}

Le colloque se propose d'apporter une nouvelle contribution à la réflexion historico-économique sur les identités nationales par la rencontre de différents spécialistes. Son but est celui d'étudier la lente apparition de l'économie comme science, de la fin du $\mathrm{xviI}^{\mathrm{e}}$ siècle à la fin du $\mathrm{xIx}^{\mathrm{e}}$ siècle, sous des formes et selon des modalités différentes tant dans les pays occidentaux qu'en Europe orientale.

Des communications seront présentées par :

M. Albertone (Istituto Universitario Orientale, Naples), A. V. Anikin (IMEMO, Moscou), M. Augello (Université de Teramo), J. Backhaus (Rijksuniversiteit, Limburg), W. J. Barber (Université de Wesleyan), M. Bianchini (Université de Parme), J. L. Cardoso (Université de Lisbonne), M. Dorigny (C.N.R.S., Paris), R. Faucci (Université de Pise), N. K. Figurovskaja (Académie des sciences, Moscou), P. Groenewegen (Université de Sidney), M. Guidi (Université de Teramo), F. Hincker (Université de Paris I), J. Jedlicki (Académie des sciences, Varsovie), G. Köver (Université de Budapest), L. Levan-Lemesle (Université de Paris I), E. Lluch (Université internationale Menéndez Pelayo), A. Masoero (Université de Venise), M. L. Pesante (Université de Turin), E. Pesciarelli (Université d'Ancône), D. I. Platonov (Université de Moscou), A. S. Skinner (Université de Glasgow), A. Teichova (Université de l'East Anglia), D. N. Winch (Université de Sussex).

Coordination : M. Albertone, A. Masoero.

Revue de synthèse : IV S. Nos 3-4, juil.-déc. 1991. 